\title{
Laser Doppler Perfusion Imaging Is Useful in the Study of Raynaud's Phenomenon and Improves the Capillaroscopic Diagnosis
}

\author{
EDOARDO ROSATO, FEDERICA BORGHESE, SIMONETTA PISARRI, and FELICE SALSANO
}

\begin{abstract}
Objective. To investigate capillary morphology and skin blood flow of dorsal hands by nailfold videocapillaroscopy (NVC) and laser Doppler perfusion imaging (LDPI), respectively, in patients with primary Raynaud's phenomenon (PRP) and systemic sclerosis (SSc) and to compare the results with those obtained in healthy controls.

Methods. The study group consisted of 142 patients with SSc, 88 patients with PRP, and 147 healthy controls. NVC was performed in all the groups examined. In patients with SSc the capillaroscopic pattern was classified as early, active, or late group pattern. A baseline skin blood flow determination of the dorsum of the subject's hands was acquired through a low-energy $670 \mathrm{~nm}$ Lisca Laser Doppler Perfusion Imager.

Results. In the healthy controls the perfusion distribution pattern was homogeneous, with a proximal-distal perfusion gradient. In patients with PRP, the perfusion distribution pattern was homogeneous, but the proximal-distal perfusion gradient was absent. Finally, in patients with SSc the perfusion distribution pattern was dyshomogeneous and a proximal-distal gradient was absent. The minimum perfusion, mean perfusion, maximum perfusion, and standard deviation, calculated as variation by means of each measurement site, were significantly different in all the groups examinated. Conclusion. NVC represents the best method to analyze microvascular damage in rheumatic diseases. LDPI improves the evaluation of vascular damage in patients with SSc. The LDPI and the capillaroscopic images fully matched the definition of the various stages of vascular digital damage in SSc. (First Release August 15 2009; J Rheumatol 2009;36:2257-63; doi:10.3899/jrheum.090187)
\end{abstract}

Key Indexing Terms: LASER DOPPLER PERFUSION IMAGING SYSTEMIC SCLEROSIS SKIN BLOOD FLOW

\section{RAYNAUD'S PHENOMENON NAILFOLD VIDEOCAPILLAROSCOPY}

The term Raynaud's phenomenon (RP) is used to describe episodic events representing vasoconstriction of the digital arteries, precapillary arterioles, and cutaneous arteriovenous shunts. It typically starts in one or several digits after exposure to the cold or a stressful situation and then spreads symmetrically to all fingers of both hands. The prevalence of RP in the general population is about 3\%-5\%. Primary Raynaud's phenomenon (PRP) is characterized by symmetric attacks, absence of tissue necrosis, ulceration or gangrene, the absence of a secondary cause, normal nailfold capillaries, a negative test for antinuclear antibodies (ANA), normal erythrocyte sedimentation rate (ESR), and normal value of C-reactive protein (CRP). Symptoms are generally mild, and the median onset age is 14 years. Conversely, a

From the Department of Clinical Medicine, Clinical Immunology Unit - Scleroderma Center, Sapienza University of Rome, Rome, Italy. E. Rosato, MD, PhD; F. Borghese, MD; S. Pisarri, MD, Professor of Internal Medicine; F. Salsano, MD, Professor of Internal Medicine, Director, Clinical Immunology Unit, Sapienza University.

Address correspondence to Prof. F. Salsano, Department of Clinical Medicine, Sapienza University, Viale dell'Università 37, 00185, Rome, Italy.E-mail: felice.salsano@uniroma1.it

Accepted for publication May 6, 2009. secondary cause is suggested when the RP onset age is over 30 years; these episodes are more intense, painful, and asymmetric, and can be associated with ischemic skin lesions. Moreover, the presence of symptoms of a connective tissue disease, specific circulating autoantibodies, and evidence of microvascular disease, detected by analysis of nailfold capillaries, can be observed ${ }^{1}$. Systemic sclerosis (SSc) is a clinically heterogeneous disorder of the connective tissue characterized by immune activation, microvascular injury, and fibrosis ${ }^{2}$. Irrespective of the classification of the disease, SSc is typically associated with $\mathrm{RP}^{3}$. RP secondary to SSc is characterized by microvascular damage and high plasma adrenomedullin and endothelin-1 levels ${ }^{4-6}$.

Capillaroscopy and testing for ANA, ESR, and CRP should be performed routinely in patients with $\mathrm{RP}^{7}$. Capillaroscopy is crucial in order to differentiate between PRP and secondary $\mathrm{RP}^{8}$. Nailfold videocapillaroscopy (NVC) represents the best method to analyze microvascular damage in rheumatic diseases. In healthy subjects, the microvascular pattern is characterized by a regular array of microvessels with large intra/interindividual variability. The presence of megacapillaries and a decreased capillary density are the hallmarks of the SSc capillary pattern, which can

Personal non-commercial use only. The Journal of Rheumatology Copyright @ 2009 . All rights reserved. 
be detected by nailfold capillaromicroscopy. Recently, 3 distinct major NVC patterns have been considered useful for assessing the appearance and the progression of sclerodermic microangiopathy: early, active, and late patterns ${ }^{9,10}$.

Several noninvasive techniques for evaluating perfusion of the hand and wrist arteries have been brought into clinical practice. Among these are color-coded duplex Doppler ultrasonography, computed tomography, and recently, magnetic resonance angiography ${ }^{11,12}$.

Laser Doppler perfusion imaging (LDPI) is a noninvasive and relatively new technique for measuring skin blood perfusion. LDPI, working according to the predicted effects of the Doppler shift of a laser beam, provides a 2-dimensional horizontal scan of the flow in a specific tissue without needing surface contact through a $670 \mathrm{~nm}$ low-energy laser beam. The recorded Doppler signal is fed to a computer via an optical isolation box, demodulated, and converted into an electrical signal. Perfusion signals are combined to form a color-coded image using a scale ranging from dark blue (lowest value) to red (highest value). The term commonly used to describe blood flow measurements by the laser Doppler technique is "flux." The flux is expressed in arbitrary perfusion units (pU) and calculated using the first moment of the power spectral density. Each measured point has a numeric $\mathrm{pU}$ between 0.00 and $10.0 \mathrm{~V}^{13,14}$.

LDPI can be useful for the detection of secondary RP, distinguishing whether the reduced blood flow is due to systemic autoimmune diseases, assessing peripheral vascular impairment in hand-arm vibration syndrome, and evaluating microvascular perfusion of morphea plaques ${ }^{15-17}$.

The aim of our study is to investigate capillaries morphology and skin blood flow of dorsal hands by NVC and LDPI, respectively, in patients with PRP and SSc and healthy controls.

\section{MATERIALS AND METHODS}

Subjects. The study group consisted of 142 patients (126 women, 16 men; mean age $55 \pm 13$ yrs) with SSc diagnosed according to the American College of Rheumatology (ACR) criteria ${ }^{18}$, admitted to the Clinical Immunology Unit of Sapienza University from January 2000 to December 2007. All patients with SSc fulfilled the ACR criteria for SSc and they were subsequently divided into limited cutaneous (lcSSc) and diffuse cutaneous (dcSSc) SSc groups, according to LeRoy, et al ${ }^{2}$. Table 1 lists the main baseline epidemiological and clinical features of the patients. Disease activity in SSc was measured using Valentini's Scleroderma Disease Activity Score $(\mathrm{SDAS})^{19}$. A disease severity index was measured by means of the Medsger Disease Severity Scale ${ }^{20}$.

Eighty-eight patients who fulfilled the PRP criteria and 147 healthy controls were also studied ${ }^{21}$. Table 2 lists the main epidemiological and clinical features of the healthy controls and patients with PRP. SSc patients and PRP patients underwent therapeutic treatment with calcium channelblockers (nifedipine $30 \mathrm{mg}$ /day). Mean duration of therapy was $13.5 \pm 12$ years for patients with SSc and $11 \pm 10$ years for PRP. Therapy was discontinued $48 \mathrm{~h}$ before examination.

Our study was approved by the local ethics committee and all patients and controls provided informed written consent. Exclusion criteria were as follows: subjects unable to give informed consent, subjects receiving actual therapy with prostacyclin analogs and/or endothelin receptor antagonists
Table 1. Systemic sclerosis (SSc) patients' epidemiological features $(\mathrm{n}=$ 142).

\section{Feature}

\begin{tabular}{lc}
\hline Sex, female/male & $126 / 16$ \\
Age, mean \pm SD yrs & $55 \pm 13$ \\
SSc duration since diagnosis, mean \pm SD yrs & $6.8 \pm 9.5$ \\
Raynaud's phenomenon duration, mean \pm SD yrs & $16 \pm 12$ \\
Subset of dcSSc/lcSSc ${ }^{2}$, no. & $56 / 86$ \\
ANA pattern, n (\%) & \\
Speckled & $24(17)$ \\
Speckled and nucleolar & $10(7)$ \\
Nucleolar & $44(31)$ \\
Centromere & $64(45)$ \\
SSc-specific autoantibody, n (\%) & \\
Anti-topo I & $44(31)$ \\
ACA & $64(45)$ \\
None & $34(24)$ \\
Disease Activity Index 19 & $2.2 \pm 1.7$ \\
Disease Severity Index ${ }^{20}$ & $4.9 \pm 2.4$ \\
Disease features, $n(\%)$ & \\
Interstitial lung disease & $64(45)$ \\
Scleroderma renal crisis & $3(2)$ \\
Myositis & $10(7)$ \\
Pulmonary arterial hypertension & $48(34)$
\end{tabular}

dcSSc: diffuse cutaneous SSc; 1cSSc: limited cutaneous SSc; ANA: antinuclear antibodies; anti-topo I: anti-topoisomerase I; ACA: anticentromere antibody.

Table 2. PRP patients' and controls' epidemiological and clinical features.

\begin{tabular}{lcc}
\hline Feature & PRP Patients $(\mathrm{n}=88)$ & Controls $(\mathrm{n}=147)$ \\
\hline Sex, female/male & $73 / 15$ & $116 / 31$ \\
Age, mean \pm SD, yrs & $38 \pm 16$ & $46 \pm 11$ \\
RP duration, mean \pm SD yrs & $16 \pm 12$ & Absent \\
ANA & Absent & Absent \\
\hline
\end{tabular}

RP: Raynaud's phenomenon; PRP: primary RP; ANA: antinuclear antibodies.

and/or phosphodiesterase 5 inhibitors, subjects with a history of uncontrolled systemic hypertension, hyperlipidemia, cardiac failure, hepatic failure or diabetes, smokers, and pregnant or breastfeeding women.

LDPI assessment. LDPI was performed in a quiet, air conditioned room (24 $\pm 0.4^{\circ} \mathrm{C}$ ) when the subjects were acclimated to the room temperature after about $20 \mathrm{~min}$. Patients and controls were not allowed to smoke or drink coffee and alcoholic beverages for $2 \mathrm{~h}$ before the examination. A baseline scan of the dorsal surface of both hands was performed in patients and controls. The skin blood flow of the dorsum of the hands was detected using a lowenergy $670 \mathrm{~nm}$ Lisca Laser Doppler Perfusion Imager (Perimed AB, Stockholm, Sweden). The scanner was placed perpendicularly $15 \mathrm{~cm}$ away from the hands according to the manufacturer's instructions. The laser sequentially scans up to $4096(64 \times 64$ pixels $)$ measurement points over the tissue under study, covering a maximal surface area of roughly $12 \times 12 \mathrm{~cm}$. Two-dimensional images of superficial tissue blood perfusion were acquired at the highest time and spatial resolution. The dorsal surface of the hands was divided into 3 regions of interest (ROI): ROI1, ROI2, and ROI3. ROI1 included the 3 fingers of the hand from the second to the fourth distal to proximal interphalangeal joint. ROI2 included the area between the proximal interphalangeal and the metacarpophalangeal joint. ROI3 included only the dorsal surface of the hand without the fingers. In each color-

Personal non-commercial use only. The Journal of Rheumatology Copyright $@$ 2009 . All rights reserved. 
Table 3. Findings of color-coded laser Doppler perfusion imaging in 3 groups of the study population.

Presence of Proximal- Presence of Homogeneous distal Perfusion Gradient, Perfusion Pattern,

\begin{tabular}{lcc} 
Group & $\mathrm{N}(\%)$ & $\mathrm{N}(\%)$ \\
\hline Controls (147) & $132(89.8)$ & $139(94.6)$ \\
PRP patients (88) & $9(10.8)$ & $82(93.2)$ \\
SSc patients (142) & $17(11.7)$ & $6(4.2)$ \\
\hline
\end{tabular}

PRP: primary Raynaud's phenomenon; SSc: systemic sclerosis.

coded image, we considered the perfusion distribution appearance and the proximal-distal perfusion gradient. Previous studies have not given a definition of this gradient and the distribution of perfusion. Since fingers are involved in patients with SSc at an early stage, we chose to evaluate the presence of a perfusion gradient between the 2 contiguous anatomical regions of the fingers. In previous studies of laser Doppler flowmetry ${ }^{14,15}$ the skin blood flow was measured in only 3 fingers (index, middle, and ring). The proximal-distal gradient is present when the mean difference in perfusion between ROI1 and ROI 2 is $>0.3 \mathrm{pU}$. For evaluation of the distribution pattern we used the color image aspect of ROI2 and ROI3. In definition of the perfusion pattern, ROI1 was excluded because in patients with SSc, the skin of this region may have digital ulcers that appear in the LDPI as hyperperfused and hypoperfused areas. In addition, for the small size of ROI1 the assessment of the distribution pattern is more difficult. Instead, in ROI2 and ROI3 the presence of very small hypoperfused and hyperperfused areas is due to microvascular damage. A homogeneous pattern was defined as a pattern showing structural uniformity of perfusion in ROI2 and ROI3, while a dyshomogeneous pattern was defined as a pattern showing the presence of very small hypoperfused and hyperperfused areas variously distributed in ROI2 and ROI3. In addition, for each image, the instrument provided the following numeric measures expressed in $\mathrm{pU}$ : minimum perfusion, mean perfusion, maximum perfusion, and standard deviation (SD). In SSc patients with sclerodactyly, LDPI was performed only when the maximal flexion of the fingers, evaluated as the point of maximum distance from the surface scan, was less than $5 \mathrm{~mm}$. In our experience, sclerodactyly with a flexion of the fingers less than $15 \mathrm{~mm}$ does not cause significant changes in the LDPI measures.

Nailfold videocapillaroscopy. The operator performed the NVC in each patient using an optical probe videocapillaroscopy unit equipped with a $100 \times$ magnification contact lens and connected to image analysis software (Pinnacle Studio Version 8, Pinnacle Systems, Mountain View, CA, USA).

Each subject was inside the building for a minimum of $15 \mathrm{~min}$ before the nailfold was examined at a room temperature of $20-22^{\circ} \mathrm{C}$. The nailfold (distal row) of the second, third, fourth, and fifth fingers was examined in each patient. The following capillaroscopic measures were considered according to previous observations: presence of enlarged and giant capillaries, hemorrhages, loss of capillaries, disorganization of the microvascular array, and capillary ramifications. According to Cutolo, et al, the patterns identified within the "SSc pattern" include the following: (1) early NVC pattern: few enlarged/giant capillaries, few capillary hemorrhages, relatively well-preserved capillary distribution, no evident loss of capillaries; (2) active NVC pattern: frequent giant capillaries, frequent capillary hemorrhages, moderate loss of capillaries, mild disorganization of the capillary architecture, absent or mild ramified capillaries; and (3) the late NVC pattern: irregular enlargement of the capillaries, few or absent giant capillaries and hemorrhages, severe loss of capillaries with extensive avascular areas, disorganization of the normal capillary array, and ramified/bushy capillaries ${ }^{9}$.

Statistical analysis. The statistical significance of the differences between the data was assessed using SPSS statistical software (version 15). All the results were expressed as mean \pm SD. The chi-squared test was used for cat- egorical variables. Student's t test was employed for numeric variables. A $\mathrm{p}$ value less than 0.05 was considered significant.

\section{RESULTS}

In healthy controls the perfusion distribution pattern was homogeneous, with a proximal-distal perfusion gradient. In the patients with PRP, the perfusion distribution pattern was homogeneous and the proximal-distal gradient was absent. Finally, in the patients with SSc the perfusion distribution pattern was dyshomogeneous and the proximal-distal gradient was absent (Figure 1).

LDPI showed a proximal-distal perfusion gradient in the $89.9 \%$ of healthy controls (132/147), $10.8 \%$ (9/88) of patients with PRP, and 11.7\% (17/142) of patients with SSc. The presence of proximal-distal perfusion gradient occurred significantly $(p<0.001)$ less often in PRP and SSc patients than in healthy controls. We did not find any significant difference in the presence of proximal-distal perfusion gradient between PRP and SSc groups.

A homogeneous perfusion distribution pattern was present in $94.6 \%$ of healthy controls and in $93.2 \%$ of patients with PRP. Conversely, the homogeneous perfusion distribution pattern was present in only $4.2 \%$ of patients with SSc. The dyshomogeneous perfusion distribution pattern appeared significantly $(\mathrm{p}<0.001)$ more often in patients with SSc than in healthy controls and patients with PRP.

In the 3 groups of the study population, the mean values of minimum perfusion, mean perfusion, and maximum perfusion were significantly different. These values, expressed as pU, were significantly different among PRP, SSc, and control groups. In the PRP group minimum perfusion, mean perfusion, and maximum perfusion were lower than in the SSc group and the control group (Table 4). In the SSc group these measures were lower than in the control group, but they were higher than in the PRP group (Table 4).

In the PRP group the SD was low (mean value 0.211) because the proximal-distal gradient was absent. Even though the proximal-distal perfusion gradient was absent, in the patients with SSc, the SD mean value $(0.270)$ was significantly $(\mathrm{p}<0.001)$ higher than in PRP patients for the presence of the dyshomogeneous perfusion distribution pattern. In the control group, the SD mean value was significantly $(\mathrm{p}<0.001)$ higher (mean value 0.307$)$ than in the other 2 groups because of the preservation of the proximaldistal perfusion gradient (Figure 2A). Table 5 shows the mean perfusion in the $3 \mathrm{ROI}$. In the healthy controls ROI 1 is better perfused than ROI2 and ROI3. In patients with thermoregulation dysfunction (PRP and SSc) ROI1 has lower values of mean perfusion than ROI2 and ROI3. Mean perfusion of ROI1 is high in healthy controls with a homogeneous distribution pattern, intermediate in SSc patients with a dyshomogeneous distribution pattern, and low in PRP patients with a homogeneous distribution pattern. In ROI2 and ROI3 mean perfusion and perfusion distribution pat-

Personal non-commercial use only. The Journal of Rheumatology Copyright @ 2009 . All rights reserved. 

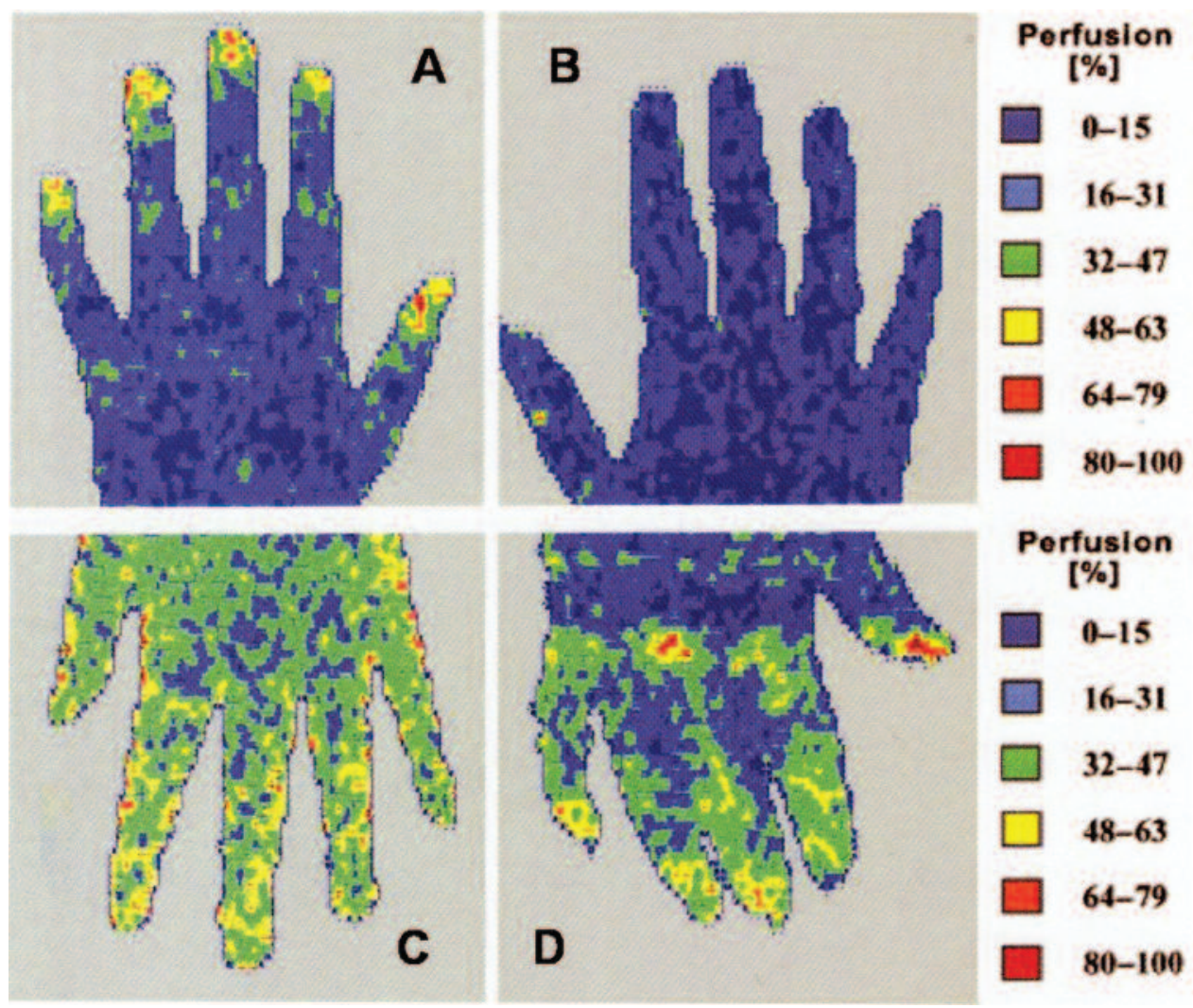

Figure 1. Color-coded laser Doppler perfusion imaging. A. Normal subject with homogeneous perfusion of the dorsal hand surface (blue) and presence of the proximal (blue in dorsal hand surface) to distal (green in 3rd phalanx) gradient. B. Primary Raynaud's phenomenon. C. Systemic sclerosis (SSc), with the early capillaroscopic pattern. D. SSc with the active pattern.

terns are similar in each group of patients. In the PRP group, ROI2 and ROI3 had a homogeneous distribution with low mean perfusion, while in the SSc group mean perfusion was low with a dyshomogeneous distribution. In healthy controls ROI2 and ROI3 had homogeneous distributions, with higher mean perfusion than the other 2 groups.

In the $3 \mathrm{SSc}$ patient capillaroscopic groups (early, active, and late pattern) the mean values of minimum perfusion, mean perfusion, and maximum perfusion were different. In the active group the mean values of these 3 measures were similar to the control group, while in the late group the values were similar to the PRP group (Table 6). In the early capillaroscopic group the mean values of minimum perfu- sion, mean perfusion, and maximum perfusion were intermediate between the other 2 capillaroscopic groups (Table 6). Also the mean SD value was different in the capillaroscopic groups of patients with SSc. Even in the case of a lack of a proximal-distal perfusion gradient, in the active capillaroscopic group of patients with SSc, the SD was equal to that in healthy controls, because in this group hyperperfused and hypoperfused areas were present. In the late capillaroscopic group, the SD was similar to that of the PRP group because of a high reduction of the perfusion within a few hyperperfused areas. In the early group, SD was intermediate between the other 2 groups for initial sclerodermic microangiopathy in the absence of hypoperfused

Table 4. Mean values, expressed as perfusion unit (pU), of minimum perfusion, mean perfusion, and maximum perfusion in 3 groups.

\begin{tabular}{lcccc}
\hline Group & $\begin{array}{c}\text { Minimum Perfusion } \\
\pm \text { SD }\end{array}$ & $\begin{array}{c}\text { Mean Perfusion } \\
\pm \text { SD }\end{array}$ & $\begin{array}{c}\text { Maximum Perfusion } \\
\pm \text { SD }\end{array}$ & $p$ \\
\hline PR patients & $0.112 \pm 0.054$ & $0.636 \pm 0.074$ & $1.793 \pm 0.528$ & $<0.0001$ \\
SSc patients & $0.167 \pm 0.070$ & $0.784 \pm 0.140$ & $2.282 \pm 0.584$ & $<0.0001$ \\
Controls & $0.196 \pm 0.064$ & $0.847 \pm 0.172$ & $2.688 \pm 0.763$ & $<0.0001$ \\
\hline
\end{tabular}

PRP: primary Raynaud's phenomenon; SSc: systemic sclerosis. 

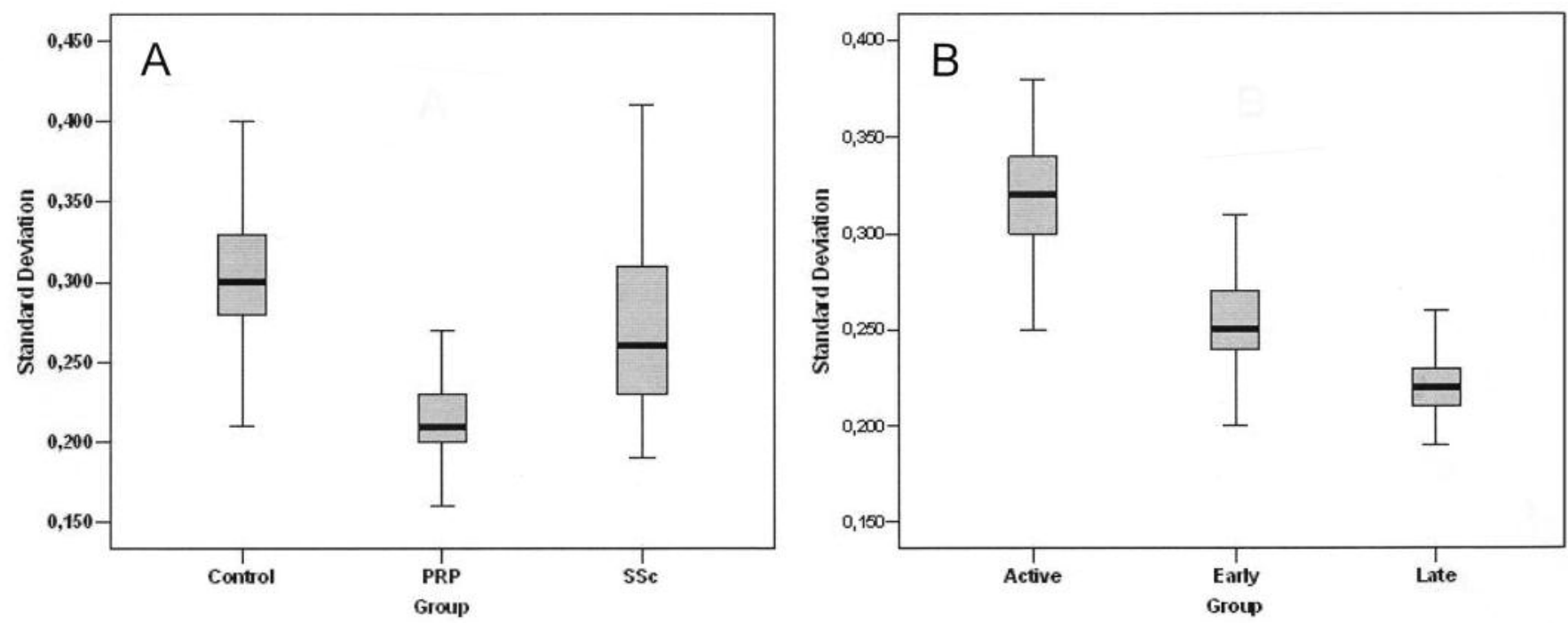

Figure 2. A. Standard deviations (SD) in 3 groups of the study population. B. SD in 3 capillaroscopic groups of patients with systemic sclerosis.

and hyperperfused areas (Figure 2B). In Table 7 we synthesized the findings of the color-coded images and mean perfusion values of LDPI. No significant differences of skin blood perfusion, measured by LDPI, were present in the 2 groups of patients with SSc (lcSSC and dcSSC).

\section{DISCUSSION}

Vascular dysfunction is one of the hallmarks of SSc and involves both the macro and microvasculature. By definition, the microcirculation is restricted to the blood flow through vessels smaller than $100 \mu \mathrm{m}$ (i.e. arterioles, capillaries, and venules). The microcirculatory flow changes with variations in concentration of blood gases, hormones, and physical factors like temperature and pressure, and is controlled by the autonomous nervous system as well. These stimuli increase or decrease the microvascular perfusion by regulating vasoconstriction or vasodilatation of the arterioles $^{22}$. Early SSc disease is mediated through microvascular dysfunction secondary to a number of factors including endothelial damage, overexpression of specific adhesion molecules, and perivascular inflammatory cell infiltration $^{23,24}$. In this process endothelial cell injury, fixed structural microvascular changes, and tissue fibrosis play a key role. Markers of endothelial activation (e.g., endothelial leukocyte-adherence molecule type 1, vascular endothelial growth factor, soluble vascular cell adhesion molecule-1, endothelin-1) are all increased in $\mathrm{SSc}^{25-27}$. In addition to changes in endothelial cell function, microvascular disease in SSc is also characterized by abnormal neurovascular control secondary to neuronal damage ${ }^{28}$. Microvascular damage

Table 5. Mean values, expressed as perfusion unit, of perfusion in 3 ROI.

\begin{tabular}{lccc}
\hline Group & $\begin{array}{c}\text { Minimum Perfusion } \\
\text { of ROI1 } \pm \text { SD }\end{array}$ & $\begin{array}{c}\text { Mean Perfusion } \\
\text { of ROI } 2 \pm \text { SD }\end{array}$ & $\begin{array}{c}\text { Maximum Perfusion } \\
\text { of ROI3 } \pm \text { SD }\end{array}$ \\
\hline RP patients & $0.641 \pm 0.076$ & $0.635 \pm 0.073$ & $0.633 \pm 0.075$ \\
SSc patients & $0.791 \pm 0.139$ & $0.792 \pm 0.138$ & $0.783 \pm 0.143$ \\
Controls & $1.542 \pm 0.238^{*}$ & $0.895 \pm 0.197$ & $0.842 \pm 0.171$ \\
\hline
\end{tabular}

* Mean perfusion of ROI1 is significantly higher than mean perfusion of ROI2 and ROI3. ROI: region of interest; RP: Raynaud's phenomenon; SSc: systemic sclerosis.

Table 6. Mean values, expressed as perfusion unit, of minimum perfusion, mean perfusion, and maximum perfusion in 3 capillaroscopic groups of patients with systemic sclerosis (SSc).

\begin{tabular}{lcccc}
\hline $\begin{array}{l}\text { SSc Patients' } \\
\text { Capillaroscopic Pattern }\end{array}$ & $\begin{array}{c}\text { Minimum Perfusion } \\
\pm \text { SD }\end{array}$ & $\begin{array}{c}\text { Mean Perfusion } \\
\pm \text { SD }\end{array}$ & $\begin{array}{c}\text { Maximum Perfusion } \\
\pm \text { SD }\end{array}$ & $p$ \\
\hline Early pattern & $0.162 \pm 0.073$ & $0.753 \pm 0.124$ & $2.165 \pm 0.523$ & $<0.0001$ \\
Active pattern & $0.192 \pm 0.083$ & $0.881 \pm 0.129$ & $2.749 \pm 0.489$ & $<0.0001$ \\
Late pattern & $0.135 \pm 0.070$ & $0.707 \pm 0.109$ & $1.845 \pm 0.306$ & $<0.0001$ \\
\hline
\end{tabular}


Table 7. Color-coded laser Doppler perfusion imaging findings and mean values of standard deviation (SD) in the study population.

\begin{tabular}{lccc}
\hline Group & $\begin{array}{c}\text { Proximal-distal } \\
\text { Gradient }\end{array}$ & $\begin{array}{c}\text { Homogeneous } \\
\text { Perfusion Pattern }\end{array}$ & SD \\
\hline Controls & Present & Present & $0.307 \pm 0.044$ \\
RP patients & Absent & Present & $0.211 \pm 0.021$ \\
SSc patients & Absent & Absent & $0.270 \pm 0.047$ \\
SSc patients' capillaroscopic pattern & & \\
Early & Absent & Absent & $0.258 \pm 0.038$ \\
Active & Absent & Absent & $0.320+0.029$ \\
Late & Absent & Absent & $0.222 \pm 0.018$ \\
\hline
\end{tabular}

RP: Raynaud's phenomenon; SSc: systemic sclerosis.

and dysfunction are involved in a number of clinical manifestations of SSc including RP, digital ulceration, pulmonary arterial hypertension, and hypertensive renal crisis. RP is often the presenting feature of SSc and it may precede skinand organ-based disease by many years. NVC, associated with the presence of serological ANA, represents the best method to clarify whether patients with RP have an underlying connective tissue disease. Several techniques have been developed to study the function of the microcirculation: transcutaneous oxygen tension, photo-plethysmography, $\mathrm{NCV}$, orthogonal polarization spectral imaging, thermal infrared imaging, and laser Doppler flowmetry (LDF). Currently, 2 variants of LDF coexist: laser Doppler perfusion monitoring (LDPM) and LDPI. The main difference between them is that LDPM uses optical fibers to carry the light to and from the tissue, while LPDI uses a scanning method with a distant light source and detector ${ }^{9,29-31}$. Previous studies have shown that LDPI is able to detect changes in SSc microvasculature, particularly when combined with NVC and thermal infrared imaging 13,32,33. In addition, LDPI has been employed in a small group of patients to evaluate skin blood flow in secondary RP, hand-arm vibration syndrome, and morphea plaques ${ }^{15-17}$.

Our study shows for the first time, in a representative group of patients, that LDPI is a useful noninvasive diagnostic technique for evaluating skin blood flow in patients with PRP and SSc. In our study population we observed that skin blood flow of the dorsal hand evaluated by LDPI was different in the 3 groups of subjects. In healthy controls, LDPI showed the presence of a proximal-distal perfusion gradient, a homogeneous perfusion pattern, and high mean value of SD. In the PRP group, LDPI demonstrated the absence of a proximal-distal perfusion gradient, a homogeneous perfusion pattern, and very low SD. Finally, in the SSc group, a proximal-distal perfusion gradient was absent, the perfusion pattern was dyshomogeneous, and the SD mean value was intermediate between the other 2 groups. In addition, the LDPI findings were different in the 3 capillaroscopic groups of patients with SSc. The early group was easily distinguishable for the absence of a proximal-distal perfusion gradient, a dyshomogeneous perfusion pattern, and a SD value intermediate between the other 2 capillaroscopic groups. The active group was characterized by the absence of a proximal-distal perfusion gradient, a dyshomogeneous perfusion pattern, and a very high SD. In SSc patients with the late pattern, a proximal-distal perfusion gradient was absent, the perfusion pattern was dyshomogeneous, and SD value was low.

We conclude that NVC still represents the best method to analyze microvascular damage in rheumatic diseases. LDPI improves the evaluation of vascular damage in patients with SSc, since we found an association of the absence of a proximal-distal perfusion gradient and a dyshomogeneous perfusion pattern only in this group of patients. In addition, the LDPI and the capillarscopic images fully matched the definitions of the various stages of vascular digital damage in SSc.

\section{REFERENCES}

1. Wigley FM. Clinical practice. Raynaud's phenomenon. N Engl J Med 2002;347:1001-8.

2. LeRoy EC, Black C, Fleischmajer R, Jablonska S, Krieg T, Medsger TA Jr, et al. Scleroderma (systemic sclerosis): classification, subsets and pathogenesis. J Rheumatol 1998;15:202-5.

3. Kahaleh MB. Vascular involvement in systemic sclerosis (SSc). Clin Exp Rheumatol 2004;22 Suppl:19-23.

4. Herrick AL. Pathogenesis of Raynaud's phenomenon. Rheumatology 2005;44:587-96.

5. Flavahan NA, Flavahan S, Mitra S, Chotani MA. The vasculopathy of Raynaud's phenomenon and scleroderma. Rheum Dis Clin North Am 2003;29:275-91.

6. Salsano F, Letizia C, Proietti M, Rossi C, Proietti AR, Rosato E, et al. Significant changes of peripheral perfusion and plasma adrenomedullin levels in acetylcysteine long term treatment of patients with sclerodermic Raynaud's phenomenon. Int J Immunopathol Pharmacol 2005;18:761-70.

7. Gayraud M. Raynaud's phenomenon. Joint Bone Spine 2007;74:1-8.

8. Maricq HR, LeRoy EC. Patterns of finger capillary abnormalities in connective tissue disease by 'wide-field' microscopy. Arthritis Rheum 1973;16:619-28.

9. Cutolo M, Sulli A, Pizzorni C, Accardo S. Nailfold videocapillaroscopy assessment of microvascular damage in systemic sclerosis. J Rheumatol 2000;27:155-60.

10. Cutolo M, Sulli A, Secchi ME, Paolino S, Pizzorni C. Nailfold capillaroscopy is useful for the diagnosis and follow-up of autoimmune rheumatic diseases. A future tool for the analysis of microvascular heart involvement? Rheumatology 2006;45 Suppl:43-6.

11. Dabich L, Bookstein JJ, Zweifler A, Zarafonetis CJ. Digital arteries in patients with scleroderma. Arteriographic and plethysmographic study. Arch Intern Med 1972;130:708-14.

12. Allanore Y, Seror R, Chevrot A, Kahan A, Drapé JL. Hand vascular involvement assessed by magnetic resonance angiography in systemic sclerosis. Arthritis Rheum 2007;56:2747-54.

13. Merla A, Di Donato L, Romani GL, Proietti M, Salsano F. Comparison of thermal infrared and laser Doppler imaging in the assessment of cutaneous tissue perfusion in scleroderma patients and healthy controls. Int J Immunopathol Pharmacol 2008;21:679-86.

Personal non-commercial use only. The Journal of Rheumatology Copyright (c) 2009. All rights reserved. 
14. Gunawardena H, Harris ND, Carmichael C, McHugh NJ. Maximum blood flow and microvascular regulatory responses in systemic sclerosis. Rheumatology 2007;46:1079-82.

15. Terada K, Miyai N, Maejima Y, Sakaguchi S, Tomura T, Yoshimasu $\mathrm{K}$, et al. Laser Doppler imaging of skin blood flow for assessing peripheral vascular impairment in hand-arm vibration syndrome. Ind Health 2007;45:309-17.

16. Szabo N, Csiki Z, Szanto A, Danko K, Szodoray P, Zeher M. Functional and morphological evaluation of hand microcirculation with nailfold capillaroscopy and laser Doppler imaging in Raynaud's and Sjögren's syndrome and poly/dermatomyositis. Scand J Rheumatol 2008;37:23-9.

17. Moore TL, Vij S, Murray AK, Bhushan M, Griffiths CE, Herrick AL. Pilot study of dual-wavelength (532 and $633 \mathrm{~nm}$ ) laser Doppler imaging and infrared thermography of morphoea. Br J Dermatol 2009;160:864-7.

18. Preliminary criteria for the classification of systemic sclerosis (scleroderma). Subcommittee for scleroderma criteria of the American Rheumatism Association Diagnostic and Therapeutic Criteria Committee. Arthritis Rheum 1980;23:581-90.

19. Valentini G, Silman AJ, Veale D. Assessment of disease activity. Clin Exp Rehumatol 2003;21 Suppl:39-41.

20. Medsger TA Jr, Bombardieri S, Czirjak L, Scorza R, Della Rossa A, Bencivelli W. Assessment of severity and prognosis. Clin Exp Rehumatol 2003;21 Suppl:42-6.

21. LeRoy EC, Medsger TA Jr. Raynaud's phenomenon: a proposal for classification. Clin Exp Rheumatol 1992;10:485-8.

22. Kellogg DL Jr. In vivo mechanisms of cutaneous vasodilation and vasoconstriction in humans during thermoregulatory challenges. J Appl Physiol 2006;100:1709-18.

23. Yamane K. Endothelin and collagen vascular disease: a review with special reference to Raynaud's phenomenon and systemic sclerosis. Intern Med 1994;33:579-82.
24. Herrick AL. Vascular function in systemic sclerosis. Curr Opin Rheumatol 2000;12:527-33.

25. Kuryliszyn-Moskal A, Klimiuk PA, Sierakowski S. Soluble adhesion molecules (sVCAM-1, sE-selectin), vascular endothelial growth factor (VEGF) and endothelin-1 in patients with systemic sclerosis: relationship to organ systemic involvement. Clin Rheumatol 2005;24:111-6.

26. Choi JJ, Min DJ, Cho ML, Min SY, Kim SJ, Lee SS, et al. Elevated vascular endothelial growth factor in systemic sclerosis. J Rheumatol 2003;30:1529-33.

27. Sato S, Hasegawa M, Takehara K. Serum levels of interleukin-6 and interleukin-10 correlate with total skin thickness score in patients with systemic sclerosis. J Dermatol Sci 2001;27:140-6.

28. Kahaleh B, Matucci-Cerinic M. Raynaud's phenomenon and scleroderma. Dysregulated neuroendothelial control of vascular tone. Arthritis Rheum 1995;38:1-14.

29. Fagrell B. Advances in microcirculation network evaluation: an update. Int J Microcirc Clin Exp 1995;15 Suppl:34-40.

30. Groner W, Winkelman JW, Harris AG, Ince C, Bouma GJ, Messmer $\mathrm{K}$, et al. Orthogonal polarization spectral imaging: a new method for study of the microcirculation. Nat Med 1999;5:1209-12.

31. Holloway GA Jr, Watkins DW. Laser Doppler measurement of cutaneous blood flow. J Invest Dermatol 1977;69:306-9.

32. Seifalian AM, Howell K, Stansby G, Jackson AE, Hamilton G, Black CM. Laser Doppler perfusion imaging: a new technique for measuring skin blood flow in rheumatology. Br J Rheumatol 1993;32:938.

33. Murray AK, Herrick AL, King TA. Laser Doppler imaging: a developing technique for application in the rheumatic diseases. Rheumatology 2004;43:1210-8. 